\title{
Spin Chain in Magnetic Field: Limitations of the Large- $N$ Mean-Field Theory
}

\author{
K. Wohlfeld ${ }^{a, b, *},{\text { Cheng-Chien } \text { Chen }^{c}, \text { M. VAn VeenendaAl }}^{c, d}$ And T.P. Devereaux ${ }^{a}$ \\ ${ }^{a}$ Stanford Institute for Materials and Energy Sciences, SLAC National Laboratory and Stanford University, \\ Menlo Park, California 94025, USA \\ ${ }^{b}$ Institute of Theoretical Physics, Faculty of Physics, University of Warsaw, Pasteura 5, PL-02093 Warszawa, Poland \\ ${ }^{c}$ Advanced Photon Source, Argonne National Laboratory, Argonne, Illinois 60439, USA \\ ${ }^{d}$ Department of Physics, Northern Illinois University, De Kalb, Illinois 60115, USA
}

\begin{abstract}
Motivated by the recent success in describing the spin and orbital spectrum of a spin-orbital chain using a large- $N$ mean-field approximation, we apply the same formalism to the case of a spin chain in the external magnetic field. It occurs that in this case, which corresponds to $N=2$ in the approximation, the large- $N$ mean-field theory cannot qualitatively reproduce the spin excitation spectra at high magnetic fields, which polarize more than $50 \%$ of the spins in the magnetic ground state. This, rather counterintuitively, shows that the physics of a spin chain can under some circumstances be regarded as more complex than the physics of a spin-orbital chain.
\end{abstract}

DOI: 10.12693/APhysPolA.127.201

PACS: 71.10.Fd, 75.10.Jm, 75.10.Pq

\section{Introduction}

Recently a number of studies discussed the collective excitations in a spin-orbital chain [1-5]. Most of them concentrated around a novel phenomenon called spinorbital separation which is present when a very strong external crystal field fully polarizes the orbital sector of the ground state [1-3]. Although this phenomenon seemed to be completely at odds with the physics present in an $\mathrm{SU}(4)$-symmetric spin-orbital chain (i.e. without external crystal field) [6], a very recent paper discusses how to unify these two seemingly different limits [5]. It occurs that a large- $N$ mean-field theory $[7,8]$ surprisingly well describes the spin and orbital spectra for any value of the crystal field and thus explains the striking evolution of the spin and orbital spectra with increasing external crystal field [5].

As a result of this recent success of the large- $N$ meanfield theory the following question arises: could such a theory be equally successful in explaining the behavior of collective spin excitations in a spin chain that is subject to external magnetic field? While this might look like as an old problem, which should have been solved long time ago, to the best of our knowledge, there exists no precise answer to this question in the literature.

\section{Definition of the problem}

Let us now be more specific. First, we define the following Hamiltonian which describes the problem of a spin chain subject to the external magnetic field $H_{z}$ :

$$
\mathcal{H}=J \sum_{\langle i j\rangle}\left(\boldsymbol{S}_{i} \boldsymbol{S}_{j}+\frac{1}{4}\right)+H_{z} \sum_{i} S_{i}^{z} .
$$

Here $J$ is the energy scale of the superexchange inter-

\footnotetext{
*corresponding author; e-mail: wohlfeld@stanford.edu
}

actions between $\mathrm{SU}(2)$-invariant spin $S=1 / 2$ operators $(\boldsymbol{S}),\langle i j\rangle$ represents a nearest-neighbor spin pair, and $H_{z}$ is the magnetic field strength.

Second, we define the transverse dynamical spin structure factor which is a good proxy for probing the nature of the collective spin excitations:

$$
S(q, \omega)=\frac{1}{\pi} \lim _{\eta \rightarrow 0} \Im\left\langle\psi\left|S_{q}^{x} \frac{1}{\omega+E_{\psi}-\mathcal{H}-\mathrm{i} \eta} S_{q}^{x}\right| \psi\right\rangle .
$$

Here $|\psi\rangle$ is the ground state of $\mathcal{H}$ with energy $E_{\psi}$, $S_{q}^{x} \equiv \sum_{j} \mathrm{e}^{\mathrm{i} q j} S_{j}^{x} / \sqrt{L}$ is the Fourier transform of the local spin operator, and $L$ is the number of lattice sites.

In what follows we calculate the dynamical spin structure factor in Eq. (2) using two distinct methods: (i) the numerically exact combined cluster perturbation theory (CPT) and exact diagonalization (ED) method, and (ii) the approximate analytical large- $N$ mean-field theory. We compare our analytical results with the numerical calculations, which have already been extensively discussed in the literature [9-13].

\section{Numerical results}

The numerical method employed in the current study, $\mathrm{CPT}+\mathrm{ED}$, is a quantum cluster approach $[14,15]$ which complements the finite-size ED simulations and therefore allows for a better visualization of the fine spectral details. The spin dynamical structure factor, Eq. (2), calculated with this method is shown in Fig. 1 for three different values of the external magnetic field $H_{z}$. When $H_{z}=0$, the ground state has short range antiferromagnetic order and the spectrum is well-known [9-11, 13]: it is mostly spanned by a two-spinon continuum and has zero modes at $q=0$ and $q=\pi$, cf. Fig. 1a. For a finite value of $H_{z}$ the ferromagnetic domains start to appear in the ground state and the positions of the zero modes shift. For instance when $H_{z} \sim 1.58 \mathrm{~J}$ half of the spins in the chain are polarized and the spectrum has zero modes 


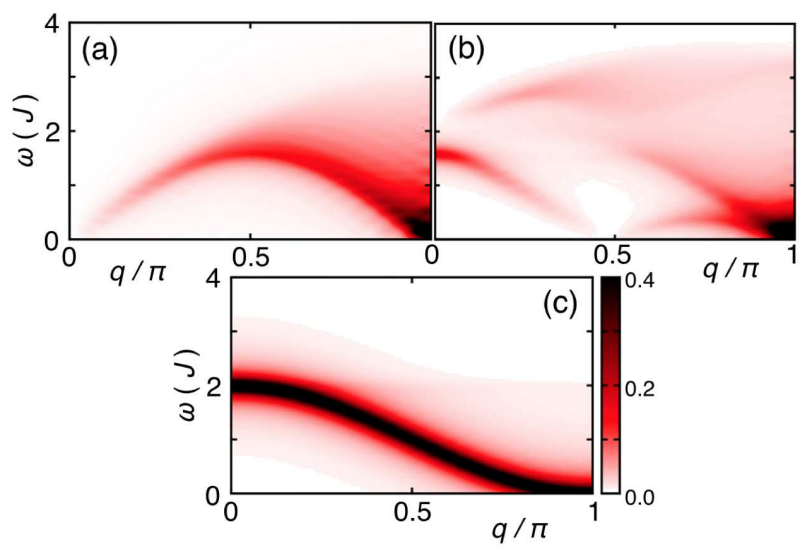

Fig. 1. Numerical results. Spin dynamical structure factors computed by $\mathrm{CPT}+\mathrm{ED}$ for a spin chain under a magnetic field $H_{z}$ : (a) $H_{z}=0$ with no spin polarization $\left(\sum_{i} S_{i}^{z} / L=0\right)$; (b) $H_{z} \sim 0.79 H_{z}^{\text {cr }}$ with half polarized spins $\left(\sum_{i} S_{i}^{z} / L=1 / 4\right) ;\left(\right.$ c) $H_{z}=H_{z}^{\text {cr }}=2.0 \mathrm{~J}$ with fully polarized spins $\left(\sum_{i} S_{i}^{z} / L=1 / 2\right)$. The ED spectra (broadened with a $0.25 J$ Lorentzian) are computed on an $L=24$ site lattice. For $H_{z}<H_{z}^{\text {cr }}$ [parts (a) and (b)], the spin spectra show fractionalized excitations with broad energy continua. When $H_{z} \geq H_{z}^{\text {cr }}$, the (ferromagnetic) ground state is fully spin-polarized; the excitations are no longer fractional, and the spectrum exhibits only a sharp, single-magnon mode [part (c)].

at $q=\pi / 2$ and $q=\pi$, cf. Fig. 1b. Nevertheless, the spectrum is still spanned by a continuum of fractional excitations. This, however, stays in contrast with the fully polarized (i.e. ferromagnetic) ground state which occurs at $H_{z}=2 J \equiv H_{z}^{\text {cr }}$. The spin dynamical structure factor is then no longer spanned by a two-spinon continuum, cf. Fig. 1c, and instead a single (magnon) branch arises. Further increasing $H_{z}$ above $H_{z}^{\text {cr }}$ leads to a gapped magnon branch.

\section{Large- $N$ mean-field theory}

Following similar steps as described in detail in Refs. [5, 16] we first map the spin model described by Eq. (1) onto a fermionic model [16]. By performing a large- $N$ mean-field decoupling and solving the selfconsistent mean-field equations, we obtain the following Hamiltonian:

$$
\mathcal{H}^{\mathrm{MF}}=\sum_{k}\left(\varepsilon_{k \uparrow} f_{k \uparrow}^{\dagger} f_{k \uparrow}+\varepsilon_{k \downarrow} f_{k \downarrow}^{\dagger} f_{k \downarrow}\right),
$$

where the fermionic bands are $\varepsilon_{k \uparrow / \downarrow}=$ $-2 J \cos \left(\delta_{k}\right) \cos k / \pi \mp J \sin \left(2 \delta_{k}\right) / \pi$ (cf. Fig. 2) with $\delta_{k}=\arcsin \left(H_{z} \pi /(2 J)\right) / 2$, subject to the constraint $\sum_{\sigma} f_{i \sigma}^{\dagger} f_{i \sigma}=1$.

We note, first, that in this approach $H_{z} \leq 2 J / \pi$, which means $\delta_{k} \leq \pi / 4$ and a maximum Fermi momentum $\delta_{k}+$ $k_{F}=3 \pi / 4$ under an applied magnetic field (since $k_{\mathrm{F}}=$ $\pi / 2$ for the fermionic mean-field theory at $\left.H_{z}=0[16]\right)$. Second, above $H_{z}>2 J / \pi$ the self-consistent mean-field solution effectively breaks down.

Next, since the maximum available Fermi momentum, $3 \pi / 4$, corresponds to just half of the spins being polarized

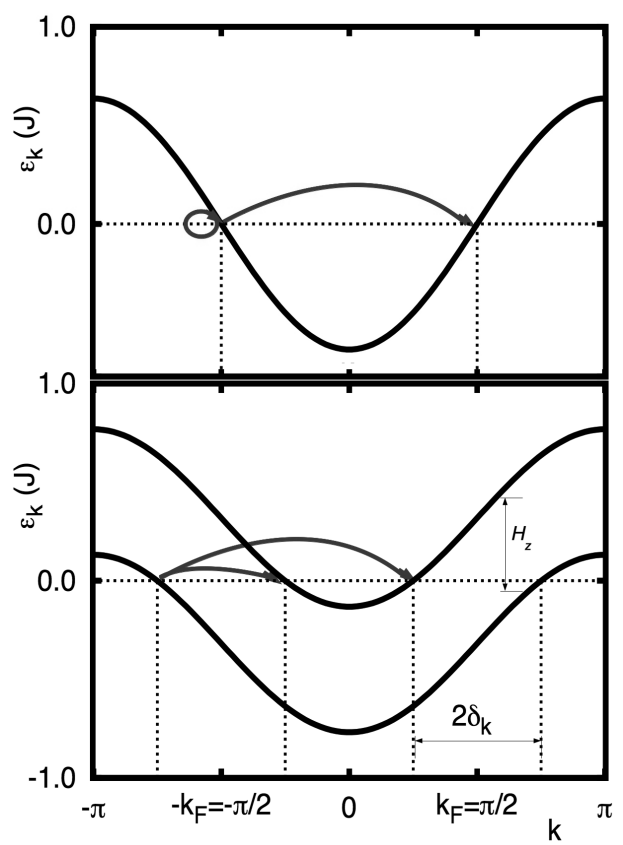

Fig. 2. Large- $N$ mean-field theory. Evolution of the mean-field fermionic bands as a function of the magnetic field at $H_{z}=0$ (top part) and $H_{z}=2 J / \pi$ (bottom part). The collective spin excitations in the meanfield picture correspond to "particle-hole" excitations of the fermions across the Fermi surface (denoted by the dotted horizontal lines). The energies of the up-spin and down-spin fermionic bands are separated by $H_{z}$, and the allowed "particle-hole" excitations change with the magnetic field accordingly. The thick arrows point to the allowed zero-energy spin excitations.

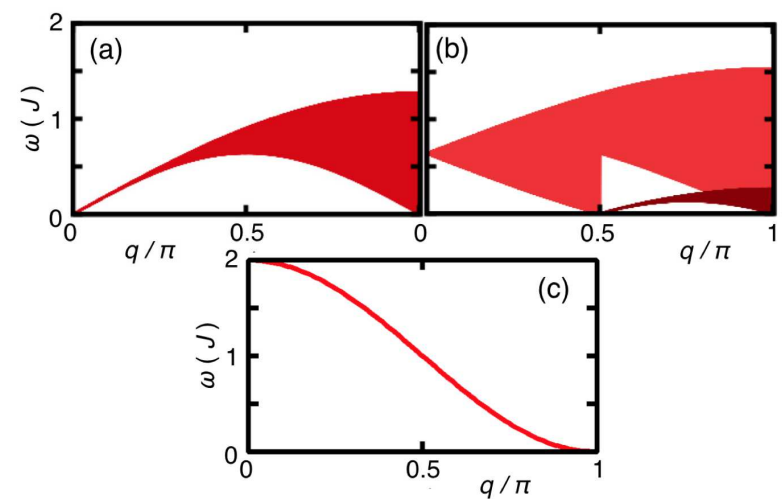

Fig. 3. Analytical results. Compact support of the spin spectra for a spin chain in a magnetic field $H_{z}$ computed by the large- $N$ mean-field theory $[(\mathrm{a})-(\mathrm{b})]$ : (a) When $H_{z}=0$, the ground state exhibits (shortrange) antiferromagnetic correlations without any spin polarization. (b) When $H_{z}=2 J / \pi$ in the mean-field calculation, i.e. when $50 \%$ of the spins are polarized in the ground state; the lighter (darker) part in the spin spectrum refers to spin-flip excitations created by spin raising (lowering) operators. (c) The exact spin wave dispersion $\omega_{q}=J(1+\cos q)$ calculated by linear spin wave theory at $H_{z}=H_{z}^{\text {cr }}$; the large- $N$ mean-field theory is not valid in this regime. 
in the ground state, this is the maximum value of polarization available in this approach. As this means that we can never fully fill one of the spin bands, i.e. reach the $\delta_{k}+k_{\mathrm{F}}=\pi$ momentum, the fermionic mean-field theory is not able to describe the fully spin-polarized ground state.

In this fermionic mean-field picture, the compact support of the spin spectrum can be calculated by

$$
\begin{gathered}
\bar{S}(q, \omega)=\sum_{k \in \mathrm{FS}, q+k \notin \mathrm{FS}} \delta\left(\omega-\varepsilon_{q+k, \uparrow}+\varepsilon_{k \downarrow}\right) \\
+\sum_{k \in \mathrm{FS}, q+k \notin \mathrm{FS}} \delta\left(\omega-\varepsilon_{q+k, \downarrow}+\varepsilon_{k \uparrow}\right),
\end{gathered}
$$

cf. Fig. 2. The evolution of the spin spectrum as a function of $H_{z}$ is shown in Fig. 3a,b. The shift of the zeroenergy modes with the magnetic field in the spin spectrum follows from the splitting of the fermionic bands in the magnetic field, cf. Fig. 2.

\section{Conclusions}

Let us first emphasize that the fermionic large- $N$ mean-field approximation is valid only when $H_{z} \leq 2 \mathrm{~J} / \pi$ (i.e. when the ground state is less than half-polarized). In this case there exists a qualitative agreement between the analytically calculated compact support and the numerically obtained spin spectrum. Nevertheless, the quantitative differences between the mean-field and the $\mathrm{CPT}+\mathrm{ED}$ results are substantial (the bandwidth of the spin excitations is ca. 2.5 smaller in the analytical approach), making the fermionic large- $N$ mean-field description less appropriate compared to that in the spinorbital model [5].

A different situation exists when $H_{z}>2 J / \pi$ (i.e. when the ground state is more than half-polarized). Here the mean-field approximation breaks down. This means that long before the critical field is reached (which fully polarizes the ground state) there exists no simple analytical approach that can be used to calculate the spin spectrum (for more complex approaches, cf. Ref. [9, 17]). It is then only when $H_{z} \geq H_{z}^{\mathrm{cr}}$ that another simple analytical approach becomes valid - the well-known linear spin-wave approximation which works well for spin ground states with long range magnetic order [11, 18]. The analytically evaluated spin spectrum, $S(q, \omega)=\delta\left(\omega-\omega_{q}\right)$ with $\omega_{q}=J(1+\cos q)$ being the magnon dispersion when $H_{z}=H_{z}^{\text {cr }}$ (cf. Fig. 3c), is then equal to the numerically calculated spin dynamical structure factor (cf. Fig. 1c).

\section{Acknowledgments}

K.W. and T.P.D. acknowledge support from the DOE-BES Division of Materials Sciences and Engineering (DMSE) under Contract No. DE-AC02-76SF00515 (Stanford/SIMES). K.W. acknowledges support from the Polish National Science Center (NCN) under Project No. 2012/04/A/ST3/00331. C.C.C. is supported by the Aneesur Rahman Postdoctoral Fellowship at Argonne National Laboratory (ANL), operated by the U.S. DOE
Contract No. DE-AC02-06CH11357. M.v.V is supported by the DOE Office of BES Award No. DEFG02-03ER46097 and the NIU Institute for Nanoscience, Engineering and Technology. This work utilized computational resources at NERSC, supported by the U.S. DOE Contract No. DE-AC02-05CH11231.

\section{References}

[1] K. Wohlfeld, M. Daghofer, S. Nishimoto, G. Khaliullin, J. van den Brink, Phys. Rev. Lett. 107, 147201 (2011).

[2] J. Schlappa, K. Wohlfeld, K.J. Zhou, M. Mourigal, M.W. Haverkort, V.N. Strocov, L. Hozoi, C. Monney, S. Nishimoto, S. Singh, A. Revcolevschi, J. Caux, L. Patthey, H.M. Rønnow, J. van den Brink, T. Schmitt, Nature 485, 82 (2012).

[3] V. Bisogni, K. Wohlfeld, S. Nishimoto, C. Monney, J. Trinckauf, K. Zhou, R. Kraus, K. Koepernik, C. Sekar, V. Strocov, B. Buechner, T. Schmitt, J. van den Brink, J. Geck, Phys. Rev. Lett. 114, 096402 (2015).

[4] W.-L. You, A.M. Oleś, P. Horsch, Phys. Rev. B 86, 094412 (2012); W. Brzezicki, J. Dziarmaga, A.M. Oleś, Phys. Rev. Lett. 112117204 (2014).

[5] C.-C. Chen, M. van Veenendaal, T.P. Devereaux, K. Wohlfeld, arXiv:1402.3255 (2014).

[6] Y.-Q. Li, M. Ma, D.-N. Shi, F.-C. Zhang, Phys. Rev. B 60, 12781 (1999).

[7] G. Baskaran, Z. Zou, P. Anderson, Solid State Commun. 63, 973 (1987).

[8] I. Affleck, J.B. Marston, Phys. Rev. B 37, 3774 (1988).

[9] G. Müller, H. Thomas, H. Beck, J.C. Bonner, Phys. Rev. B 24, 1429 (1981).

[10] D.C. Dender, P.R. Hammar, D.H. Reich, C. Broholm, G. Aeppli, Phys. Rev. Lett. 79, 1750 (1997).

[11] S. Grossjohann, W. Brenig, Phys. Rev. B 79, 094409 (2009).

[12] M. Kohno, Phys. Rev. Lett. 102, 037203 (2009).

[13] M. Mourigal, M. Enderle, A. Klöpperpieper, J.-S. Caux, A. Stunault, H.M. Rønnow, Nat. Phys. 9, 435 (2013).

[14] D. Sénéchal, D. Perez, M. Pioro-Ladrière, Phys. Rev. Lett. 84, 522 (2000).

[15] T. Maier, M. Jarrell, T. Pruschke, M.H. Hettler, Rev. Mod. Phys. 77, 1027 (2005).

[16] M. Kenzelmann, C.D. Batista, Y. Chen, C. Broholm, D.H. Reich, S. Park, Y. Qiu, Phys. Rev. B 71, 094411 (2005).

[17] M. Karbach, D. Biegel, G. Müller, Phys. Rev. B 66 , 054405 (2002).

[18] A. Auerbach, Interacting Electrons and Quantum Magnetism, Springer, New York 1994. 